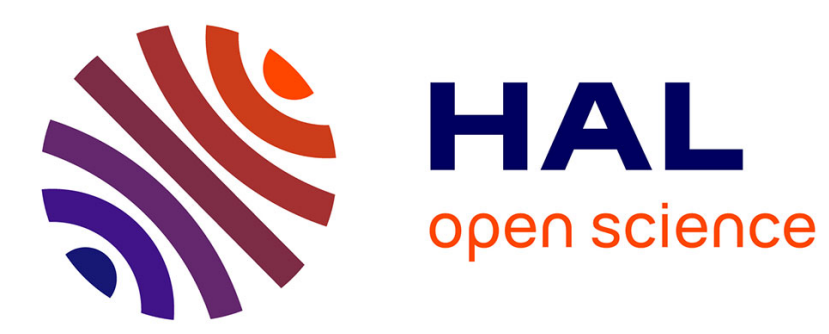

\title{
Les élèves, analyseurs du dispositif partenarial d'une classe de quatrième externée
}

\author{
Claire de Saint Martin
}

\section{To cite this version:}

Claire de Saint Martin. Les élèves, analyseurs du dispositif partenarial d'une classe de quatrième externée. Agora débats/jeunesses, 2018, 80 (3), pp.25-40. 10.3917/agora.080.0025 . hal-03163230

\section{HAL Id: hal-03163230 \\ https://hal.science/hal-03163230}

Submitted on 9 Mar 2021

HAL is a multi-disciplinary open access archive for the deposit and dissemination of scientific research documents, whether they are published or not. The documents may come from teaching and research institutions in France or abroad, or from public or private research centers.
L'archive ouverte pluridisciplinaire HAL, est destinée au dépôt et à la diffusion de documents scientifiques de niveau recherche, publiés ou non, émanant des établissements d'enseignement et de recherche français ou étrangers, des laboratoires publics ou privés. 


\title{
LES ÉLÈVES, ANALYSEURS DU DISPOSITIF PARTENARIAL D’UNE CLASSE DE QUATRIÈME EXTERNÉE
}

Claire de Saint Martin

\author{
Presses de Sciences Po (P.F.N.S.P.) | «Agora débats/jeunesses »
}

$2018 / 3 \mathrm{~N}^{\circ} 80$ | pages 25 à 40

ISSN 1268-5666

ISBN 9782724635331

Article disponible en ligne à l'adresse :

https://www.cairn.info/revue-agora-debats-jeunesses-2018-3-page-25.htm

\section{Pour citer cet article :}

Claire de Saint Martin, « Les élèves, analyseurs du dispositif partenarial d'une classe de quatrième externée », Agora débats/jeunesses 2018/3 ( $N^{\circ}$ 80), p. 25-40. DOI 10.3917/agora.080.0025

Distribution électronique Cairn.info pour Presses de Sciences Po (P.F.N.S.P.).

(C) Presses de Sciences Po (P.F.N.S.P.). Tous droits réservés pour tous pays.

La reproduction ou représentation de cet article, notamment par photocopie, n'est autorisée que dans les limites des conditions générales d'utilisation du site ou, le cas échéant, des conditions générales de la licence souscrite par votre établissement. Toute autre reproduction ou représentation, en tout ou partie, sous quelque forme et de quelque manière que ce soit, est interdite sauf accord préalable et écrit de l'éditeur, en dehors des cas prévus par la législation en vigueur en France. Il est précisé que son stockage dans une base de données est également interdit. 


\section{Les élèves, analyseurs du dispositif partenarial d'une classe de quatrième externée}

Claire de Saint Martin

\section{INTRODUCTION}

En mars 2015, vingt-deux élèves en quatrième au collège de L'île-Rousse, en Corse, ont bénéficié d'une classe d'expression artistique et culturelle pendant cinq jours. Ils devaient écrire des nouvelles fantastiques à partir de la découverte de la microrégion montagneuse du Giussani, située audessus de L'Île-Rousse. Ce projet était conduit par Anna, leur professeure de français, Fiona, leur professeure de corse, ainsi que par Rémy, comédien et dramaturge, et Natale, ancien instituteur du village, tous deux intervenants de l'association d'éducation populaire ARIA (Association des rencontres internationales artistiques). Rémy devait accompagner les élèves dans leur processus d'écriture à partir des promenades organisées par Natale dans les environs du village. À travers ce séjour, plusieurs objectifs étaient poursuivis : permettre aux élèves de vivre une expérience collective, modifier leur rapport à l'écriture, identifié comme difficile par Anna, leur faire découvrir le territoire dans lequel ils vivent.

L'éducation populaire (EP) a une longue tradition d'éducation par l'art : « Au sens large, l'éducation populaire désigne les initiatives éducatives qui se déroulent en son nom, principalement en dehors de l'école, à l'origine dans un souci de démocratisation des savoirs, et qui sont animés par une intention plus ou moins forte de rénovation des pédagogies scolaires. » (Morvan, 2017, p. 107.) L'ARIA, créée par le comédien Robin Renucci, reprend ces objectifs et affirme le théâtre comme lieu du politique : «Apprendre de l'autre, grandir, se tenir debout, s'exprimer, respirer, créer, voilà les maîtres-mots qui nous guident dans la construction de cette aventure et que nous continuons à inventer ${ }^{1}$. » L'association «s'adresse à tous ceux qui se retrouvent dans une démarche d'éducation populaire, consistant à donner à chacun les moyens de son émancipation individuelle et collective ${ }^{2}$. » Elle défend l'idée 
Les élèves, analyseurs du dispositif partenarial

d'une classe de quatrième externée

de l'éducation et de la formation par « la réalisation par l'art ${ }^{3}$ » et organise tout au long de l'année des stages de théâtre et des projets artistiques à l'adresse des écoles, des collèges et des lycées, sous la conduite de Léa 4 , administratrice de production et d'éducation artistique à l'ARIA. L'association dispose à Pioggiola et Olmi-Cappella, deux villages du Giussani, d'un théâtre et d'un bâtiment autorisant des séjours en internat. Ses pratiques pédagogiques permettent « de travailler à la mise en place d'échanges réciproques de savoirs et de pratiques » (Bonnefon, 2006, p. 46). Anna collabore régulièrement avec l'association dont elle partage les valeurs et l'ambition.

La recherche menée lors de ce séjour visait à étudier les effets de ce dernier sur les élèves. Le cadre théorique de la socio-clinique institutionnelle (Monceau, 2012 ; Saint Martin de, 2014) a introduit une autre partenaire, la chercheuse, mais a accordé aussi une place centrale aux élèves dans le processus de recherche, les considérant comme des cochercheurs (Beauchesne et al., 2005). Cet article rend compte des effets de l'implication des jeunes dans ce dispositif partenarial complexe. Comment répondent-ils à ce projet ? Comment leur participation active dans le processus de recherche définitelle les effets produits sur eux par ce séjour au regard des objectifs fixés par les différents intervenants?

Après avoir explicité le contexte de la recherche, j'exposerai les effets du projet identifiés par les élèves. La troisième partie de cet article analysera comment la production de connaissances par ces élèves a pointé les contradictions de cette « institution incertaine » (Lyet, 2015 et 2016).

\section{LE CONTEXTE DE LA RECHERCHE}

\section{La socio-clinique institutionnelle}

La socio-clinique institutionnelle, issue de l'analyse institutionnelle (Lourau, 1970), vise à mettre au jour les contradictions de l'institution, définie « comme un entrelacement vivant de règles, de normes et de résistances à celles-ci » (Monceau, Soulière, 2017). L'institution est une dynamique qui n'existe qu'en actes, par les rapports entre les différents acteurs qui la composent. Elle comporte des dimensions organisationnelles, idéologiques, libidinales : les affects. René Lourau (1970) la définit par trois moments opérant dans un mouvement permanent : les règles établies, l'institué, sont constamment bousculées par des forces instituantes; l'institutionnalisation constitue le moment où l'institué absorbe l'instituant, se modifiant alors et créant de nouvelles règles. «En résumé, l'institution est la somme des forces qui la

3. Renucci, entretien, le 4 décembre 2015.

4. Tous les noms ont été anonymisés. 
traversent. » (Pilotti, 2016, p. 120.) Toute institution est traversée par des non-dits, des contradictions, dont le dévoilement, par l'analyse institutionnelle, contribue à produire des connaissances à visées opératoires et scientifiques. Nous verrons comment les élèves ont constitué une force instituante dans le cadre institué de la classe externée.

Cette démarche qualitative prend en compte « la dynamique institutionnelle dans ses analyses localisées » (Monceau, 2003, p. 13). La question de l'échantillon ne se pose pas ici : toute situation dit quelque chose de l'institution, par l'analyse de l'implication de ses acteurs. Tout individu entretient des rapports avec l'institution dans laquelle il se trouve, y est impliqué de fait. Il s'agit alors de comprendre comment. Les analyseurs, «léléments perturbateurs de l'institué) qui en éclairent les rouages et les enjeux » (Monceau, Soulière, 2017), bousculent l'institué et obligent chacun à expliciter ses rapports à l'institution, en révélant ainsi les contradictions. « Naturels ou construits, les analyseurs permettent de lire les implications. » (Rougerie, 2017, p. 168.) L'analyseur construit est introduit par le chercheur. Quand je propose aux élèves de réfléchir sur les raisons pour lesquelles leur professeur a initié ce séjour, cet analyseur les incite à expliciter leur rapport à l'écriture dans le cadre scolaire. L'analyseur naturel « est imprévisible et surgit spontanément dans les situations de crise » (Guillier, 2001, p. 16). Nous verrons comment l'absence de notation au cours du séjour a constitué un analyseur naturel de la négativité du collège. L'analyse institutionnelle vise à dévoiler « le négatif non intégré, non récupéré, non dépassé, par l'intermédiaire des analyseurs qui provoquent l'impensé de la structure sociale à se manifester » (Lourau, 1973, p. 26). La négativité, en analyse institutionnelle, désigne les tensions, les résistances. "Cette négativité est à l'œuvre dans toutes les institutions éducatives, sur tous les lieux et dans tous les moments du savoir institué. » (Lourau, 1970, p. 250-251.) Son étude permet la compréhension du processus institutionnel à l'œuvre.

La socio-clinique institutionnelle est une démarche collective. Le savoir produit est celui « produit par les acteurs eux-mêmes, et en situation de travail analytique [...] » (Lapassade, Lourau, 1974, p. 178). Ici, la recherche s'inscrit dans un dispositif partenarial spécifique engageant des acteurs distincts : les professeurs, les intervenants, les élèves et la chercheuse.

\section{Un dispositif partenarial spécifique}

Le dispositif s'est construit en transversalité à trois institutions : un collège, un laboratoire universitaire et une association d'EP, dans le cadre d'une « recherche avec »: «La recherche avec se pratique et s'acquiert dans l'expérimentation de la collaboration vue comme confrontation à l'altérité. » (Monceau, Soulière, 2017.) 
Les élèves, analyseurs du dispositif partenarial

d'une classe de quatrième externée

L'ARIA travaille à l'élaboration des projets en étroite collaboration avec les enseignants. «C'est complètement à la carte. Il y a un premier échange avec l'enseignant et, en fonction de sa demande, on essaie de trouver l'intervenant qui correspond le mieux et le contenu qui lui convient le mieux. La démarche vient du prof ${ }^{5}$. » C'est Anna qui a proposé ce projet d'écriture. Elle souhaitait que «le départ de l'écriture, l'envie d'écrire se fassent à partir du lieu et des lieu $x^{6} »$. Des intervenants partageant les valeurs de l'association sont contactés selon les projets :

«C'est des intervenants qui ont tous été stagiaires ici. Je pense que c'est bien, parce qu'on se comprend. On parle le même langage, on est sur les mêmes valeurs la plupart du temps, complètement même. » (Léa, entretien individuel, 19 décembre 2014.)

Léa a proposé alors à Rémy, qui avait déjà travaillé avec l'association, de tenir les ateliers d'écriture à partir des visites conduites par Natale.

Comédienne amateure ayant fait plusieurs stages à l'ARIA et membre de l'association, je connaissais déjà Léa, Rémy et Natale. J'avais rencontré Anna au cours d'un stage. Elle s'était intéressée au dispositif que j'avais créé avec des élèves dans le cadre de mon doctorat (Saint Martin, 2014). Quand elle a construit ce projet, elle a pensé m'y associer pour étudier l'appropriation du dispositif par les élèves. Léa s'est montrée immédiatement réceptive à notre proposition. Le regard du chercheur sur les pratiques collaboratives l'intéressait.

Anna, Léa, Rémy et Natale ont élaboré ensemble le projet. L’idée centrale en était la découverte des lieux comme moteur de l'écriture de nouvelles fantastiques. Le choix de la classe a été défini par Anna en fonction de l'inscription au programme de quatrième de la nouvelle fantastique au XIX $X^{e}$ siècle : «Le format de la nouvelle me semblait bien convenir au projet d'écriture dans ce cadre-là ${ }^{7}$. » Sous la conduite de Natale, les élèves découvriraient les environs le matin ; l'après-midi, Rémy dirigerait les ateliers d'écriture. Une lecture publique des textes conclurait le séjour. Des temps seraient consacrés à la recherche collaborative avec les élèves, dans un dispositif que j'exposerai plus loin.

J'ai participé à la vie du groupe durant ces cinq jours, du 16 au 20 mars 2015, assistant à toutes les activités, aux concertations entre adultes. J'ai également mené plusieurs entretiens individuels avec Anna, Léa, Rémy, Fiona, Robin ainsi que deux restitutions collectives au mois de juin, l'une avec les collégiens, l'autre avec Anna et Léa au sein de l'association. Dans ce dispositif, j'étais considérée comme une intervenante auprès des

5. Léa, entretien individuel, 19 décembre 2014.

6. Anna, entretien, 26 février 2015.

7. Anna, entretien, 26 février 2015. 
élèves, au même titre que les autres adultes, avec ma propre fonction, celle de chercheuse.

Ainsi, le cadre d'action était institué par le dispositif partenarial. « Ces dispositifs reposent sur l'engagement d'individus et sur l'émergence de collectifs locaux. » (Lyet, 2016, p. 18.) Ils nécessitent une relation de confiance entre les partenaires. Ici, les adultes se connaissaient et partagent les valeurs défendues par l'ARIA, créant une confiance entre tous. Nous verrons que les élèves ont pu pointer les effets contradictoires de cette relation de confiance. Philippe Lyet (2015) parle d' « institution incertaine » pour désigner la dynamique instituante de ce type de dispositif qui se heurte à la faiblesse de l'institué. Dans notre cas, c'est à travers la place des élèves dans le processus de recherche qu'ont pu être repérées les contradictions du dispositif.

\section{La place des élèves dans le dispositif}

Les élèves avaient une place particulière dans le dispositif. Ils en étaient les destinataires, puisqu'il s'est construit sur la base de l'analyse de la professeure de français quant à leurs difficultés à entrer dans un processus d'écriture. La pratique créative de l'écriture dans le cadre de l'association devait leur permettre de modifier leur rapport à l'écriture.

Cependant, le processus de recherche plaçait les élèves au centre de cette pratique créative. J'appréhende les pratiques professionnelles non à partir des professionnels eux-mêmes, mais à partir de leurs destinataires. La socioclinique institutionnelle vise la réappropriation de la puissance d'agir des individus, le développement de la réflexivité autorisant les individus à la compréhension de leurs rapports à l'institution (Cultiaux, Fugier, 2017, p. 32). Dans ce contexte, «la prise en compte de l'expérience des personnes dans la mise en place de pratiques qui les touchent et les concernent devient incontournable » (Monceau, Soulière, 2017). J'ai organisé avec les élèves trois séances de réflexion collective au cours du séjour, par demi-groupes de onze. Si j'introduisais ces séances par une question ou une observation, le déroulement de la réflexion leur appartenait. En effet, le chercheur ici n'est pas au centre du dispositif mais favorise l'émergence d'une discussion entre pairs (Saint Martin, 2014). Je ne posais pas d'hypothèses, mais j'accompagnais un travail collectif qui produit des connaissances en situation. « Le défi est donc de saisir des évolutions, du mouvement et de la transformation en train de se faire. » (Monceau, 2013, p. 91). Ainsi associés à la recherche, « les enfants ne sont plus seulement des enquêtés, mais des cochercheurs » (Saint Martin, 2014, p. 103.) « Ils sont non pas des sujets de recherche, mais des cochercheurs engagés dans une démarche de collaboration à la construction de savoirs pertinents pour l'avancement de connaissances scientifiques et professionnelles. » (Beauchesne et al., 2005, p. 384.) En analysant la situation qu'ils vivent, « ils participent alors à la production de savoir. » (Pilotti, 2016, p. 110.) 
Les élèves, analyseurs du dispositif partenarial

d'une classe de quatrième externée

À la fin des séances, je restituais leur analyse aux adultes. Voyons quels effets a produits cette analyse quant aux objectifs pédagogiques.

\section{LES EFFETS DU DISPOSITIF AU REGARD DES OBJECTIFS PÉDAGOGIQUES}

Je restitue l'analyse collective des élèves qui a fait l'objet d'un consensus au terme des différentes séances : si des positions singulières sont apparues, elles sont restées marginales et concernent peu l'objet de cet article. C'est pourquoi j'emploie majoritairement le terme collectif « les élèves ».

\section{Le vivre-ensemble}

La découverte d'un autre lieu de vie devait permettre d'instaurer de nouvelles relations entre les élèves. Ceux-ci ont tout de suite perçu cette dimension, envisageant la classe externée comme une occasion d' « encore resserrer les liens », de « s'éclater un peu aussi puisqu'on est ensemble ${ }^{8}$. » Le séjour devait autoriser une plus grande connaissance des uns et des autres qui a effectivement changé les regards : « Comme

Le fait d'être en dehors du collège, de vivre une expérience collective inédite pour la majorité favorise une entraide entre pairs qui est aussi liée à de nouvelles relations avec Anna et Fiona. y'a des personnes, on change d'opinion sur elles ${ }^{9}$. » La distanciation par rapport à la vie du collège, la suppression de la routine qui lui est associée, l'absence de concurrence qu'elle instaure apaisent les tensions relationnelles. «On s'engueule toujours entre nous. Alors que, comme ça, tous ensemble, depuis ce matin, y'a personne qui s'est engueulé avec personne ${ }^{10}$. »

Cette perception ne résiste pas à la réalité d'une vie commune que la plupart éprouvent pour la première fois et à l'existence de conflits durant le séjour. Mais en juin, les élèves maintiennent leur discours : "C'était bien, parce qu'on était tous ensemble ${ }^{11}$. » Ils soulignent qu'entre eux, ils parlent «beaucoup plus qu'en début d'année ${ }^{12}$ ». Si le retour à la vie collégienne a réinstitué de la compétition entre eux, le séjour a institutionnalisé de nouvelles relations et modalités de travail : «Des fois, on travaille quand même ensemble, des fois on s'aide et tout ${ }^{13}$. »
8. Séance de réflexion collective, 16 mars 2015.
9. Ibid.
10. Ibid.
11. Restitution collective, 3 juin 2015.
12. Ibid
13. Ibid 
Au fil du séjour, je note une évolution des postures des élèves.

« J'entends Alizée corriger les fautes d'accord de Thierry, en le questionnant pour l'amener lui-même à nommer le plus-que-parfait... Finalement, le fait de ne pas intervenir conduit les élèves eux-mêmes à se corriger mutuellement quand la lecture des textes des autres est empêchée par les fautes. » (Journal de recherche, mercredi 18 mars 2015, après-midi.)

Le fait d'être en dehors du collège, de vivre une expérience collective inédite pour la majorité favorise une entraide entre pairs qui est aussi liée à de nouvelles relations avec Anna et Fiona :

« On a plus de haine quand on est au collège envers un professeur que quand on est en sortie. » (Séance de réflexion collective, 18 mars 2015.)

«Moi, je trouve que c'est bien, parce qu'elles nous aident en fait, elles sont là derrière nous. » (Séance de réflexion collective, 18 mars 2015.)

Ces nouvelles relations conduisent les élèves à un nouveau rapport à l'écriture.

\section{Le rapport à l'écriture des collégiens}

Le rapport à l'écriture est ici défini comme un « ensemble touffu, complexe, fait d'une multitude de variables entremêlées » (Barré-de Miniac, 2000, p. 13). Il comporte des dimensions personnelles, individuelles, mais aussi collectives, « c'est-à-dire liées à l'appartenance de chacun de nous à des groupes de plus en plus importants » (ibid.). Dans le cadre de la socioclinique institutionnelle, sont aussi pris en compte les aspects institutionnels et organisationnels qui inscrivent ce rapport à l'écriture dans le rapport aux institutions (Samson et al., 2016).

Les élèves reconnaissent la valeur de l'intervention de Rémy par rapport aux cours habituellement dispensés au collège («C'était mieux expliqué14. »). Ils ont particulièrement apprécié la séance en demi-groupe du jeudi après-midi, qui s'est déroulée en extérieur. Antone, l'un des plus réfractaires à l'activité d'écriture, précise l'importance du changement de lieu : « Déjà, simplement, on a appris qu'on pouvait apprendre tout en s'amusant ${ }^{15}$. » La prise en charge des activités par un intervenant extérieur, la restitution de mon analyse des premières séances ont déterminé un changement fondamental dans la posture d'Anna qui a cessé de porter son attention sur l'orthographe et la syntaxe. On retrouve ici l'analyse de Pascale Garnier (2003) qui souligne l'importance de l'intervention d'un tiers dans l'enrichissement de l'activité professorale. Cette posture a institutionnalisé un autre rapport à l'écriture des élèves :

14. Restitution collective, 3 juin 2015.

15. Séance de réflexion collective, 18 mars 2015. 
Les élèves, analyseurs du dispositif partenarial

d'une classe de quatrième externée

«Voilà, mais on n'est pas obligé de bien mettre le verbe à la bonne personne, enfin, si on écrit avec " $n t$ " au lieu de " $t$ ", ben c'est pas grave, alors qu'en rédaction, et bien c'est important au collège. » (Séance de réflexion collective, 18 mars 2015.)

Surtout, l'écriture, ne faisant pas l'objet d'une évaluation scolaire, se distingue d'une activité scolaire normative. Les élèves soulignent l'importance de l'absence de notation qui les enferme dans un système entravant leur créativité.

Matteu : Ce qui me force à bien travailler, moi, c'est les notes, d'avoir la meilleure possible.

Enquêtrice : Et ça, le fait de ne pas être noté, ça te libère ici ?

Matteu : On décompresse.

Aline : On peut oser des choses qu'on n'oserait peut-être pas en rédaction.

Romain : Ah, ça c'est vrai, la peur de se tromper.

Sylvia : On peut faire des histoires qu'on n'oserait même pas rendre.

(Réflexion collective, groupe 2, mercredi 18 mars.)

Les élèves sont unanimes pour noter une évolution positive de leur rapport à l'écriture. Lors de la première séance de réflexion collective, ils attendaient que ce travail libère leur imagination qu'ils reconnaissaient comme faible. Cependant, au cours des différentes activités, ils étaient davantage en difficulté dans les exercices faisant appel à des souvenirs réels que dans les exercices d'imagination. Je leur ai proposé alors une séance, imprévue, le mercredi soir et leur ai renvoyé cette contradiction. Ils ont contesté mon analyse :

Karine : Ben, j'ai dit que moi, enfin c'est pas mon cas, ce que vous avez dit sur l'imagination, moi j'ai pas dit ça.

Jean : Moi aussi, dès que je commence à écrire, je m'arrête plus.

Anna : Oui, j'ai toujours des difficultés et là c'est sorti tout seul, c'est la première fois.

[...]

Luc : Moi, j'ai plusieurs idées, mais je ne sais pas comment les mettre au propre, savoir écrire.

Théo : Ouais, voilà, on sait pas comment écrire, comment les expliquer.

Enquêtrice : Et qu'est-ce qui vous bloque dans le passage à l'écrit?

Luc : Comment faire les phrases.

(Entretien collectif élèves, groupe 1, mercredi 18 mars 2015.)

En juin, ils ont déclaré que «ça a marché ». Dans ce collège, les élèves de quatrième passent les épreuves du brevet blanc en janvier et en avril. Tous approuvent Aline quand elle exprime sa perception de l'épreuve de français :

«Par exemple, au brevet, la rédaction, j'ai pas eu de problèmes, alors qu'avant, je passais quatre ans à trouver un sujet, des idées et tout ça. » (Restitution collective, 3 juin 2015.)

Anna a donc atteint un de ses objectifs : non seulement la majorité des élèves ont acquis un rapport plus aisé à l'écriture, mais ils ont pris plaisir 
à écrire. Nous verrons plus loin que cet effet est parfois limité au temps de la classe externée.

\section{La découverte du territoire}

L'un des objectifs d'Anna était de pallier la méconnaissance des collégiens sur leur territoire :

«Si tu habites île-Rousse, tu ne connais pas forcément Olmi-Capella. Même cette relation-là, avec le patrimoine géographique, culturel, je suis sûre que pour beaucoup d'élèves ça va être une découverte. Je trouve qu'ils ont de moins en moins de connaissances du territoire sur lequel ils vivent. » (Anna, entretien individuel, 26 février 2015.)

La classe est composée d'élèves habitant L'île-Rousse et ses environs. Cependant, selon Anna, «très peu sortent de leur périmètre d'habitation ${ }^{16}$ ». Le Giussani est situé à une heure de route. Lors du trajet nous y conduisant, j'ai effectivement été surprise par les réactions des élèves :

«Après Belgodère, à la vue d'un filet d'eau coulant à flanc de montagne, une s'exclame "oh, de l'eau". Puis, quand trois vaches traversent la route "oh, une vache!" Puis "oh, la neige !". Dans le virage avant d'arriver vers Vallica, à la vue du Sao Parteu "oh, c'est beau !" Ces réflexions me surprennent. Elles me semblent celles de citadins plus que d'enfants vivant dans un milieu rural et confirment ce que m'a dit Marie : les enfants vivent sur un territoire très restreint. » (Journal de recherche, lundi 16 mars 2015.)

Cependant, la météo défavorable des deux premiers jours n'a pas permis la découverte du patrimoine. L'activité d'écriture a donc débuté sur d'autres critères. Les deux promenades, tardives sur le temps du séjour lle mercredi après-midi et le jeudi matin), n'ont pas été exploitées. Les élèves se sont alors interrogés sur leur lien avec le projet d'écriture : «Voilà, ça nous apprend des trucs et tout ça, mais je vois pas où était le rapport avec l'écriture ${ }^{17}$. » Les promenades sont considérées comme de simples sorties : « Ça nous a fait prendre l'air, parce que sinon, on était tout le temps enfermés ${ }^{18}$. 》 L'environnement a été inséré à un moment où les élèves étaient déjà bien avancés dans l'écriture de leur texte. (« Moi, personnellement, j'ai pas vu le rapport entre l'église, un mausolée et le stage d'écriture qu'on a fait ici ${ }^{19}$. ») Aucun n'a utilisé les promenades dans cette écriture.

Ainsi, les contretemps météorologiques n'ont pas permis aux élèves une nouvelle appréhension du territoire et l'éveil de leur curiosité. Nous verrons

16. Anna, entretien individuel, 26 février 2015.

17. Séance de réflexion collective du 18 mars 2015.

18. Idem.

19. Idem. 
Les élèves, analyseurs du dispositif partenarial

d'une classe de quatrième externée

plus loin que leurs protestations sont également un analyseur des non-dits de cette institution incertaine.

\section{L'émancipation}

Robin Renucci insiste sur la mission de l'EP qui est de permettre à chaque individu de devenir « auteur de soi-même ${ }^{20}$ », ici par l'éducation par l'art. Libérés de la contrainte du collège, n'étant plus soumis à sa compétition permanente et à l'obligation de travailler pour des notes, les élèves ont libéré leur créativité en découvrant le plaisir d'écrire, au-delà de leurs difficultés. Lors de la séance collective du vendredi 18 mars, Letizia dit qu'elle a accepté d'écrire ici alors qu'elle refuse de prendre un stylo au collège. Quand je reviens en juin, j'apprends que, de retour au collège, elle a de nouveau refusé d'écrire et qu'elle est absente depuis un mois. L'effet du séjour est

Libérés de la contrainte du collège, n'étant plus soumis à sa compétition permanente et à l'obligation de travailler pour des notes, les élèves ont libéré leur créativité en découvrant le plaisir d'écrire, au-delà de leurs difficultés. resté circonscrit au temps de la classe externée et Letizia n'a pas supporté le retour à l'institué du collège.

Tout au long du séjour, les élèves se sont libérés du regard des adultes en s'entraidant de plus en plus. L'écriture est devenue plus autonome et affirmée. Le vendredi matin, Auriane a décidé de ne pas suivre les consignes de Rémy pour la fin de son texte, parce qu'elle trouvait sa proposition plus juste.

Le mercredi soir, certains se sont plaints de ne pas écrire collectivement.

Alizée : Si, c'est bien de pouvoir parler avec nos camarades pour aider. Jean : De partager des trucs, c'est bien.

Luc : C'est vraiment un avantage de parler avec son camarade je pense, pour avancer tous les deux, petit à petit.

Jean : Quand on parle, certes c'est bien, mais on est trop nombreux, y'a trop d'idées. Moi ce que j'aurais préféré c'est, genre, on se met par groupes de trois et on essaie de formuler un texte.

(Entretien collectif, groupe 1, mercredi 18 mars 2015.)

Au cours des échanges, les élèves décident d’aller voir Rémy pour lui demander une séance d'écriture collective, qu'il organise.

Pierre Roche insiste sur la validité de l'accompagnement groupal dans le «processus de réappropriation de la puissance d'agir » (Roche, 2017, p. 130). Ici, c'est bien le lieu collectif de l'EP, les séances de réflexion collective qui ont permis aux élèves de devenir une force instituante en affirmant leur désir. En « prenant le pouvoir », ils se libèrent des normes scolaires et entrevoient

20. Robin Renucci, entretien, 4 décembre 2015. 
un autre possible. La classe externée a rempli sa mission, au-delà de la seule activité d'écriture.

\section{LES ÉLĖVES PRODUCTEURS DE CONNAISSANCES : LES CONTRADICTIONS DES INSTITUTIONS}

\section{La négativité du collège}

L'analyse des élèves met en exergue la négativité du collège, dont le système de notation constitue un analyseur. Le fonctionnement scolaire institue un rapport à l'écriture douloureux en raison de la hiérarchie des notes : « $\mathrm{Au}$ collège, il y a toujours une compétition au niveau des notes ${ }^{21}$. 》 Cela conditionne leur rapport au savoir. Transparaît ici la négativité du collège, où l'écriture est instrumentalisée au profit de l'apprentissage du code écrit : «Et on s'est pas occupés des fautes d'orthographe, alors qu'en cours on s'en serait préoccupés plus que de l'histoire ${ }^{22}$. »

Le discours des élèves sur l'évolution de leurs relations au sein de la classe, à la suite du séjour, donne à voir combien la compétitivité instituée influe sur ces relations. Les élèves pointent également comment la normativité induite par le système de notation définit les relations entre les professeurs et les élèves, par une posture pédagogique des professeurs différente durant le séjour :

«Puis, aussi, on peut mieux discuter avec les professeurs. C'est pas que dans le sens "j'ai combien à mon contrôle", que "le devoir, il a été corrigé". » (Séance de réflexion collective, 18 mars 2015 .)

Leur discours rejoint celui des adultes qui reconnaissent l'incapacité de l'école à poursuivre certains objectifs :

« Je ne vois pas comment à l'intérieur d'un établissement, sans un partenariat avec les associations de l'EP, tu peux faire quelque chose d'autre que ce tu fais dans le cadre de tes cours. » (Anna, entretien, 21 mars 2015.)

Dans le discours d'Anna transparaît la négativité du système scolaire dont la normativité empêche certains apprentissages. De même, Rémy ${ }^{23}$ note l'importance du lieu de l'EP :

«Je pense que le premier truc, c'est d'arriver à casser l'école ; ils ne sont pas à l'école. » (Journal de recherche, lundi 16 mars 2015, après-midi.)

21. Séance de réflexion collective, 16 mars 2015.

22. Séance de réflexion collective, 18 mars 2015.

23. Rémy, entretien, 26 février 2016. 
Les élèves, analyseurs du dispositif partenarial

d'une classe de quatrième externée

Rémy précise qu' « il n'y a pas de bien et de mal, on n'est pas à cet endroitlà ». Il parle de bienveillance dans l'accompagnement de la production d'écriture.

En percevant les spécificités pédagogiques instituées par le lieu de l’EP, les élèves dévoilent la négativité du collège.

\section{Les non-dits institutionnels}

Les non-dits institutionnels s'ancrent d'abord dans la réalité matérielle, concrète. La météo a déterminé de constantes modifications dans l'emploi du temps. Rémy a consacré plusieurs séances à une activité théâtrale non prévue, centrale les deux premiers jours. Les séances de réflexion collective ont été longuement consacrées à des protestations à ce propos : «Après, au bout d'un moment, quand on en fait trop, trop, trop, c'est sûr que ça devient saoulant ${ }^{24}$. » Les élèves ne font pas le lien entre cette activité et le travail d'écriture : «À la base, on est venus là pour un projet d'écriture, on fait que du théâtre ${ }^{25}$. » La restitution publique du travail prend la forme d'une mise en espace théâtralisée qui suscite l'étonnement de Léa :

«Où est l'essence du début ? Qu'est-ce qui s'est passé pendant ces cinq jours ? Parce que moi, j'arrive, je vois une restitution qui est bien mais n'est pas celle prévue. » (Léa, restitution, 3 juin.)

Ces protestations sont un analyseur des non-dits institutionnels. Léa a engagé Rémy sans en référer à Anna qui voulait assurer les ateliers d'écriture. Si cette dernière a accepté ce changement, il a eu des effets sur le projet. Anna a proposé le thème de la nouvelle fantastique. Or, pour Rémy, « prendre un sujet scolaire, même si on est à l'EP, même si on a un intervenant extérieur, on se raccroche à un programme scolaire et on les [les élèves] remet dans des problématiques d'école ${ }^{26}$. » Il a proposé aux élèves des jeux d'écriture qui ont facilité leur adhésion mais ont occulté le thème du fantastique. Les élèves ont ensuite eu du mal à insérer cette dimension dans leur texte, réécriture d'un des écrits produits au cours de ces jeux.

« Le fait de passer à un type d'écriture plus narrative met certains élèves en difficulté : jusqu'à présent, les jeux d'écriture se déroulaient en cinq minutes et n'exigeaient pas de longs développements. L'introduction du fantastique est aussi difficile. Auriane me dit : "On ne se perd pas dans une tente !" » (Journal de recherche, mercredi 18 mars, 2015, après-midi.)

L'association travaille avec des personnes qui partagent ses valeurs et fonde ainsi chaque partenariat sur la confiance. Les contraintes matérielles,

24. Séance de réflexion collective, 18 mars 2015.

25. Ibid.

26. Rémy, entretien, 26 février 2016. 
météorologiques, la distribution des tâches entre adultes, les non-dits ont généré des tensions non exprimées entre les intervenants. "La confiance en l'engagement de l'autre ne peut se vérifier qu'à la pratique. » (Lyet, 2016, p. 21.) Si deux réunions ont été tenues durant le séjour, aucun partenaire n'a explicité ses désaccords quant au travail de l'autre. Ces silences ont institutionnalisé un échange des places des partenaires et la scolarisation du lieu de l'EP que les élèves ont très rapidement perçus.

\section{La scolarisation du lieu de l'EP}

Si les élèves ont noté le déplacement de posture de leurs professeurs, ils insistent aussi sur celui de Rémy qui a pris progressivement la posture de professeur, en contradiction avec son propre discours. Ils dénoncent sa rigueur qui renvoie à l'école :

«Même, je trouve que les professeurs sont moins stricts que Rémy l'était. » (Séance de réflexion collective, 20 mars 2015.)

«Il disait qu'il était comédien ou écrivain, mais en tout cas, il faisait prof. » (Restitution collective, 3 juin 2015.)

« Et aussi, quand il nous explique au début, ça va parce qu'on a un peu d'inspiration, mais le problème c'est qu'après il nous répète et répète et réexplique et répète et après c'est bon, l'inspiration, elle est partie. » (Séance de réflexion collective, 18 mars 2015.)

Si les élèves ont noté le déplacement de posture de leurs professeurs, ils insistent aussi sur celui de Rémy qui a pris progressivement la posture de professeur, en contradiction avec son propre discours.

Ces reproches renvoient en creux à une situation scolaire où l'enseignant capte la parole, empêchant celle des élèves.

Le fait que la classe externée se déroule sur le territoire de l'EP accorde la primauté à l'intervenant. «Alors, quand chacun se crispe sur une logique identitaire, une représentation stéréotypée du "métier de l'autre" se cristallise, qui vaut aussi pour "l'autre" tout court, puisque cette réalité est incarnée par un sujet. » (Filiod, 2010, p. 87.) Les professeures, sur le lieu de l'EP, laissent à Rémy la seule décision des activités et ce parfois au prix du projet initial. Ses transformations n'ont pas été l'objet de discussions. "Le pari de la confiance est confirmé quand ce qui se passe correspond à ce qui était prévu. » (Lyet, 2016, p. 21.) Les non-dits institutionnels conduisent Rémy à prendre la «place du prof ${ }^{27} »$.

De plus, s'il a été informé de ma présence et l'a acceptée, il n'a pas été consulté à ce sujet. Cette imposition a produit des effets sur la conduite des ateliers.

27. Séance de réflexion collective, 18 mars 2015. 
Les élèves, analyseurs du dispositif partenarial

d'une classe de quatrième externée

« Chaque fois que Rémy parle, il me regarde à la fin de son intervention, comme s'il guettait ma réaction. J'essaie de rester impassible, mais je sens que ma présence le perturbe. II ne peut pas l'oublier. » (Journal de recherche, mercredi 18 mars 2015, après-midi.)

Quand j'aborde cette question avec lui, il me répond cependant : « Ça ne m’a pas dérangé et ça a même été intéressant parce qu'il y avait une circulation entre les enseignants, toi et $\mathrm{moi}^{28}$. » L'attitude de Rémy est néanmoins un analyseur de ma propre posture : en portant une attention particulière aux élèves dans le processus de recherche et étant assurée de la commande passée par Anna et Léa, j'ai oublié de considérer Rémy dans la mise en place du dispositif.

Ces postures aboutissent à une contradiction : la scolarisation du lieu de l'EP. La première séance d'écriture a débuté par une interrogation orale sur la définition du fantastique, renvoyant les élèves à une situation scolaire. Cette institutionnalisation scolaire de l'EP s'observe matériellement. Le lieu rappelle une salle de classe : installées face à Rémy, les tables ne permettaient pas aux élèves d'expérimenter d'autres postures scripturales.

« Cette forme d'institution qui est mouvement avant d'être structure et où l'instituant est prépondérant sur l'institué est donc extrêmement sensible à toute variation et à tout changement. » (Lyet, 2015.) Ces changements produisent ici la scolarisation du lieu de l'EP, marquée par un échange des places des adultes, échange consenti parce que non discuté.

\section{CONCLUSION}

L'analyse collective des élèves a mis au jour les contradictions de cette institution incertaine. Les valeurs partagées des différents acteurs ne suffisent pas à faire aboutir un projet commun, ici considérablement transformé : l'écriture ne s'est pas faite à partir de la découverte du patrimoine du Giussani, le thème du fantastique et la forme de la nouvelle ont été occultés, l'activité théâtrale a été introduite. Cependant, malgré les dissensions entre adultes, ces mêmes valeurs ont permis de réaliser la plupart des objectifs pédagogiques fixés et ont contribué à émanciper les élèves des normes scolaires, les autorisant à prendre le pouvoir. Si l'institution incertaine se caractérise par sa fragilité, elle rend aussi possible le partenariat par la mise en place d'un dispositif spécifique.

En devenant cochercheurs, les élèves ont apporté à ce dispositif des éléments de compréhension essentiels. En ce sens, la recherche rejoint le principe de démocratisation défendue par l'EP. « Cette démocratisation implique aussi de construire la recherche en fonction d'intérêts communs de compréhension et de transformation, élaborés et partagés entre les chercheurs et les participants. » (Monceau, Soulière, 2017.)

28. Rémy, entretien, 26 février 2016. 
Les élèves, analyseurs du dispositif partenarial d'une classe de quatrième externée

\section{BIBLIOGRAPHIE}

BarRÉ-De Miniac C., 2000, Le rapport à l'écriture. Aspects théoriques et didactiques, Presses universitaires du Septentrion, Villeneuve-d'Ascq.

Beauchesne A., Garant C., Dumoulin M.-J., 2005, « Le rôle de cochercheur chez le partenaire du milieu scolaire dans les recherches collaboratives », Revue des sciences de l'éducation, n² 2, vol. 31, p. 377-395.

Bonnefon G., 2006, Penser l'éducation populaire. Humanisme et démocratie, Lyon, Chronique sociale.

Cultiaux J., Fugier P., 2017, Face à la domination : dévoiler, résister, s'émanciper, Paris, L'Harmattan.

FILIOD J.-P., 2010, « Des malentendus, bien entendu ! Partenariat, tensions et innovations discrètes dans un dispositif de "résidences d'artistes en école maternelle" ", Les sciences de l'éducation - Pour l'Ėre nouvelle, n 4, vol. 43, p. 77-94.

GARnier P., 2003, Faire la classe à plusieurs. Maîtres et partenariats à l'école élémentaire, Rennes, Presses universitaires de Rennes.

GUILLIER D., 2001, «Petite histoire de l'analyseur argent », Les cahiers de l'implication, $n^{\circ}$ 5, hiver 2001-02, p. 9-25.

LaPAssade G., Lourau R., 1974, Clefs pour la sociologie, Paris, Seghers.

Lourau R., 1970, L'analyse institutionnelle, Paris, Minuit.

Lourau R, 1973, «Analyse institutionnelle et question politique », L'homme et la société, n²9-30, p. 21-34.

LyET P., 2015, « La coopération partenariale : une institution incertaine », Paris, Biennale internationale de l'éducation, de la formation et des pratiques professionnelles « Coopérer? ».

LYET P., 2016, « Les transactions partenariales dans le travail social : des institutions incertaines », Pensée plurielle, n 43, p. 15-22.

Monceau G., 2003, « Pratiques socianalytiques et socio-clinique institutionnelle », L'homme et la société, n 147, p. 11-33.

Monceau G. (dir.), 2012, L'analyse institutionnelle des pratiques. Une socio-clinique des tourments institutionnels au Brésil et en France, Paris, L'Harmattan.

Monceau G., 2013, «Effets d'une pratique clinique de recherche », in Kohn R. C. (coord.), Pour une démarche clinique engagée, Paris, L'Harmattan, p. 91-103.

Monceau G., Soulière M., 2017, « Mener la recherche avec les sujets concernés : comment et pour quels résultats ? », Éducation et socialisation [en ligne], $\mathrm{n}^{\circ} 45$. 
Les élèves, analyseurs du dispositif partenarial

d'une classe de quatrième externée

Morvan A., 2017, «Épreuve d'éducation populaire politique au Pavé. Retour critique sur une pratique de récits de vie ». Agora débats/ jeunesses, $n^{\circ} 76$, p. 107-118.

Pilotti A., 2016, Masseurs-kinésithérapeutes salariés à l'ère numérique. Ce que leurs usages du courrier électronique disent de leur professionnalisation, Thèse de doctorat en sciences de l'éducation, Université de CergyPontoise.

Roche P., 2017, «Dominations, résistances et subjectivation au travail », in Cultiaux J., Fugier P., Face à la domination : dévoiler, résister, s'émanciper, Paris, L'Harmattan.

ROUgerie C., 2017, « L'accueil du chercheur dans une recherche collaborative », Phronesis, n 1-2, vol. 6, p. 166-176.

Saint Martin C. DE, 2014, Que disent les élèves CLIS 1 de leur(s) place(s) dans l'école ? Un empan liminal, Thèse de doctorat en sciences de l'éducation, Université de Cergy-Pontoise.

Samson D., Saint Martin C. De, Monceau G., 2016, « Perception étudiante de la commande d'écriture de mémoires et rapport à l'écriture », Nouveaux cahiers de la recherche en éducation, $\mathrm{n}^{\circ} 2$, vol. 19, p. 73-94.

\section{L'AUTEURE}

\section{Claire de Saint Martin}

clairesm1709ahotmail.fr

Maîtresse de conférences en sciences de l'éducation, laboratoire École, mutations, apprentissages (EMA), ESPE, université de Cergy Pontoise.

Thèmes de recherches : la scolarisation des élèves en situation de handicap ; la liminalité ; l'éducation inclusive ; le partenariat éducation nationale/éducation populaire ; les recherches « avec ».

\section{A notamment publié}

SAINT MARTIN C. DE, 2016, «L'empan liminal, un outil conceptuel pour penser la scolarisation des élèves de Clis 1 », La nouvelle revue de l'adaptation et de la scolarisation, $n^{\circ} 73$, p. 239-252.

SaINT MARTIN C. DE, 2017, «La place des élèves des classes spécialisées dans l'école élémentaire : un analyseur de leur construction identitaire », Éducation et socialisation. Les Cahiers du CERFEE [en ligne], $\mathrm{n}^{\circ} 44$.

SaINT Martin C. DE, Queiroz D., 2017, « Chercher avec des enfants : la nécessité du désapprentissage », Sociétés et jeunesses en difficulté [en ligne], n 18. 